\title{
Horizons/Théâtre
}

Revue d'études théâtrales

8-9| 2016

Théâtres du geste, du jeu et de la voix

\section{Les jeux de la poésie et du hasard (Sardaigne)}

\section{Maria Manca}

\section{(2) OpenEdition}

Journals

Édition électronique

URL : https://journals.openedition.org/ht/844

DOI : $10.4000 /$ ht. 844

ISSN : 2678-5420

Éditeur

Presses universitaires de Bordeaux

Édition imprimée

Date de publication : 31 décembre 2016

Pagination : 146-160

ISSN : 2261-4591

\section{Référence électronique}

Maria Manca, «Les jeux de la poésie et du hasard (Sardaigne) », Horizons/Théâtre [En ligne], 8-9 | 2016, mis en ligne le 01 janvier 2018, consulté le 18 mai 2022. URL : http://journals.openedition.org/ht/844 ; DOI : https://doi.org/10.4000/ht.844

\section{(c) $(1) \odot$}

La revue Horizons/Théâtre est mise à disposition selon les termes de la Licence Creative Commons Attribution - Pas d'Utilisation Commerciale - Pas de Modification 4.0 International. 


\section{MARIA MANCA}

Maria Manca est maître de conférence en Ethnopoétique à l'université Paris Diderot. Elle a une double formation littéraire (agrégée des universités) et ethnologique (doctorat à Paris X-Nanterre). Ses domaines de recherche sont l'anthropologie de la parole dans l'aire méditerranéenne, les joutes poétiques et plus largement la poésie chantée en performance. Son approche pragmatique de la poésie porte une attention particulière à la voix, au geste, à l'interaction et au contexte d'énonciation. Elle étudie aussi les poésies urbaines (rap et slam) à la fois dans leur aspect social, poétique et performatif. Ses principales publications sont : La Poésie pour répondre au hasard. Une approche anthropologique des joutes poétiques de Sardaigne, Paris, Ed. MSH / CNRS, 2009 ; La Voix actée. Pour une nouvelle ethnopoétique (éd.), Paris, Kimé, 2010 ; " D'un rythme à l'autre » (éd.), Cahiers de Littérature Orale n ${ }^{\circ} 73-74$, Paris, Publications Langues $O^{\prime}$, 2013

Mail : maria.manca@free.fr

Résumé : La Sardaigne traditionnelle n'offre pas d'exemple de théâtre à proprement parler. Mais lors des fêtes sont pratiqués des jeux poétiques avec le hasard, comme le maju (jeu de mai) et la gara poetica (joute poétique), qui à leur manière constituent des "spectacles». Ces jeux ont en commun de convoquer le hasard et de jouer avec lui en suivant les règles d'un game. Mais pour répondre au hasard, ils se présentent aussi sous la forme d'un play qui construit un temps et un espace, entretient une tension dramatique et distribue des rôles, met en scène des paroles poétiques chantées. L'observation de ces deux pratiques dans

Abstract: Traditional Sardinia does not afford examples of regular theatre. But, during festivals, poetical games involving chance are practiced, like maju (the May game) and gara poetica (poetical jousting), which, in a way, represent "shows ». These games have in common the fact that they invite chance and play with it while following the rules of a game. But, in order to respond to chance, they also show up as a sort of play, which constructs a time and a space, keeps up a dramatic tension, casts parts, and stages sung poetical words. Observing these two practices in their performance and their way of functioning will lead to studying how, leur performance et leur fonctionnement amènera à étudier comment, sans être du théâtre, ces jeux de poésie et de hasard engagent, chacun à sa manière, toute une communauté participante qui se met ellemême en scène et en jeu à travers ce que l'on pourrait appeler un « théâtre de soi ».

Mots-clés : jeu, hasard, poésie, théâtre, Sardaigne

without being a theatre proper, these poetry and chance games, each in its own way, commit an entire participating community which stages itself and brings itself into play through what could be called " self-theatre ".

KEYWORDS: game, chance, poetry, theatre, Sardinia

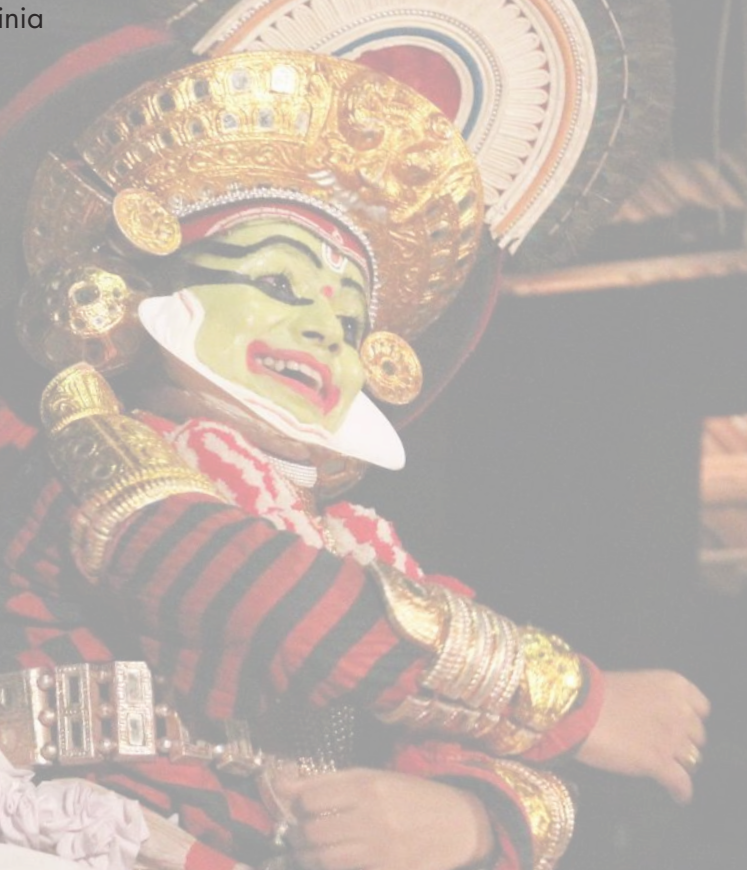




\section{Les jeux de la poésie et du hasard (Sardaigne)}

Dans la Sardaigne de tradition orale, on ne trouve pas d'exemple de théâtre à proprement parler. Les écrits des voyageurs de la fin du XVIII ${ }^{e}$ siècle et du début du XIX ${ }^{\mathrm{e}}$ ne relèvent sur l'île aucune manifestation de type « théâtral » au sens que prend communément ce mot aujourd'hui ${ }^{1}$.

En revanche, ils décrivent des pratiques de poésie, de chant, de musique et de danse comme des événements spectaculaires et festifs très répandus et suivis par un public de passionnés. Selon leurs témoignages et ceux des observateurs jusqu'aux années 1950, ces manifestations constitueraient - avec le carnaval - les seuls divertissements de la population rurale. Certaines de ces pratiques ont encore cours aujourd'hui et conservent une dimension de spectacle, mais dans un sens particulier. En effet, leurs performances prennent souvent la forme d'un jeu, non seulement entre les concurrents mais aussi avec le hasard qui s'y trouve invité.

Deux jeux de ce type seront étudiés ici. Le premier, appelé maju (jeu de mai) n'est plus pratiqué de nos jours, alors que le deuxième, la gara poetica (joute poétique) est encore en vigueur. Le maju est une cérémonie divinatoire où chaque participant se voit attribuer un sort suite à l'extraction d'objets dans un panier. La gara poetica consiste pour plusieurs poètes improvisateurs à débattre sur des thèmes opposés qu'ils tirent au sort dans une casquette. Dans les deux cas, le hasard est interrogé à travers un tirage au sort et les réponses qui lui sont apportées sont faites en poésie chantée.

Il est intéressant de remarquer que dans ces jeux la dimension de lutte ou game est étroitement liée à celle de fiction ou play. En effet, la confrontation qu'entretiennent le hasard et la poésie se donne à voir et à entendre à travers une certaine mise en scène. Mais la nature et la fonction de ce type de spectacle sont bien particulières. Ces jeux ne sont pas vécus comme des représentations théâtrales, mais des événements engageant toute une communauté à travers un « spectacle total » en train de se faire. 


\section{Jouer avec le hasard}

Le maju et la gara poetica sont d'abord des jeux avec le hasard au sens de game : lutte, compétition, qui comporte des règles et induit des statégies. Dans ces jeux, le hasard est convoqué, sollicité, subi ou affronté.

\section{Une divination}

Le maju est un jeu cérémoniel de distribution des sorts, qui était pratiqué dans la petite ville d'Ozieri au nord de la Sardaigne. Il est observé au XVIII $^{\mathrm{e}}$ siècle par Matteo $\mathrm{Madao}^{2}$, puis au XIX ${ }^{\mathrm{e}}$ par Alberto de La Marmora ${ }^{3}$ et Filippo Valla $^{4}$. Rosa Losengo ${ }^{5}$ en a recueilli des témoignages jusqu'au milieu du $\mathrm{Xx}^{\mathrm{e}}$ siècle alors qu'il n'était plus pratiqué mais que ses vers étaient encore chantés. À partir de toutes ces données, le jeu a été analysé par Alberto Mario Cirese $^{6}$ dans un article datant de 1964, qui a servi de base à ce travail.

Voici la description du maju: un groupe de jeunes filles (parfois avec des garçons) s'assoit par terre en formant un cercle au milieu duquel est placé un panier couvert, contenant des objets ou gages personnels qui appartiennent à chacun des participants (dé, couteau, chapelet, bouton). Un jeune enfant («innocent ») assis près du panier tire un à un ces gages au hasard, et avant l'extraction de chaque objet, une jeune femme (ou un chœur) qui compose le cercle chante une strophe, tantôt de bon augure ou de bénédiction, tantôt de mauvais augure ou de malédiction, jusqu'à épuisement des objets. Ainsi est annoncé à leur propriétaire incognito un sort, tantôt heureux et tantôt malheureux, suivant une stricte alternance des deux. De sorte qu'une moitié des personnes présentes est bien traitée par le sort et l'autre mal traitée, sans qu'il y ait de protestation.

Le hasard, matérialisé ici par la « pioche » d'objets directement reliés à leurs propriétaires, attribue un sort immédiat et irrévocable, incontestable et incontesté, une sorte de destin subi pour une année. En l'occurrence, le tirage au sort est un tirage des sorts où le hasard prédit à chacun son avenir. La divination comporte une dimension à la fois individuelle puisque chaque participant se voit attribuer un avenir bon ou mauvais, et collective puisqu'elle touche la totalité des participants. « Le jeu à la fois assigne individuellement et partage collectivement les sorts $\gg$ dit Cirese ${ }^{7}$. Et il ajoute que du fait du numerus clausus des joueurs, l'ensemble du groupe reçoit un nombre égal de bons et de mauvais sorts ; ce qui revient à attribuer une destinée individuelle liée à la destinée du groupe entier : « le tirage au sort assigne à chacun sa destinée et partage le bien et le mal entre tous ». Dans un article ultérieur qui interroge l'idéologie de ce jeu, Cirese $^{8}$ précise que la fatalité y est vécue comme juste et nécessaire : c'est mors tua vita mea (« ta mort contre ma 
vie $\gg$ ) d'après la conception d'une société ayant un nombre de biens limités qui doivent être équitablement partagés.

\section{Une compétition}

La gara poetica ${ }^{9}$ aussi est un jeu ${ }^{10}$, non plus de hasard mais avec le hasard. Il est encore en vigueur en Sardaigne rurale et se présente comme une compétition (c'est le sens du mot «gara $\gg$ ) dont il faut sortir vainqueur. Le jeu consiste, pour deux (ou trois) poètes, à s'affronter en improvisant à partir de thèmes inscrits sur des papiers tirés au sort dans une casquette. Ces thèmes sont en opposition les uns avec les autres : « Riche et Pauvre », « Force et Ruse », « Épouse riche, Épouse intelligente, Épouse belle ». Certains sont considérés comme « forts », d'autres comme « faibles » selon qu'ils sont jugés plus ou moins riches et faciles à traiter. Tour à tour et suivant là aussi une rigoureuse alternance, chaque poète chante une strophe pour défendre son propre thème contre celui de son collègue, en construisant avec lui une problématique pour le débat. Les poètes improvisent donc un nombre égal de strophes en joutant. Ce développement se clôt par une reconciliation des poètes et une synthèse des thèmes qui affirme leur égale importance et leur complémentarité. Les poètes disent : «Il faut de tout pour faire un monde », de même que dans le maju une quantité égale de bien et de mal est nécessaire à la communauté.

Dans la gara, le hasard est matérialisé par le tirage au sort des papiers devant le public. Mais en réalité il est plus complexe et se manifeste à plusieurs niveaux. Il est d'abord « préparé » par le comité de la fête du village, qui choisit les thèmes à débattre et les inscrit sur des petits papiers ; le hasard est aussi constitué des nombreux aléas de l'improvisation : conditions matérielles d'une joute nocturne en plein air (chaleur, vent, bruit), niveau de compétence et attention variables du public, bonne ou mauvaise «inspiration » que les poètes appellent muta bona et muta mala (de mutare, changer), qualité de la strophe du collègue à laquelle il faut répondre du tac au tac.

\section{Jeu de hasard/jeu avec le hasard}

Enfin, à la différence des participants au maju, les poètes ne subissent pas exactement le hasard. Certes, ne connaissant pas les thèmes à l'avance, ils ne peuvent ni les refuser ni les changer. Ils les acceptent, comme ils disent dans leur première strophe : «Je suis heureux du thème [appelé tema ou sorte, sort] que m'a donné la Déesse aux yeux bandés $\gg$. Et ils poursuivent : « Le sort qui m'est échu (m'est toccadu), je l'accepte et m'en réjouis/Et maintenant je dois (mi toccat) le défendre ». Mais il est remarquable que le verbe toccare prend 
ici des sens différents et reliés : il signifie 1) « toucher » matériellement (les papiers), 2) « échoir » (le sort, le hasard), 3) « devoir » (obligation, destin). Et à travers ces glissements sémantiques, le poète transforme insensiblement un hasard subi en destin accepté, tel un défi à relever : celui d'être le temps d'une joute un « Homme d'honneur » contre un « Homme d'argent », un « Quémandeur » contre un « Voleur », quelqu'un qui préfére vivre de « Pain », plutôt que d'《 Amour » ou de « Fantaisie ». Le tirage au sort oblige le poète à accepter son thème, mais pour échapper à cette contrainte, il va se l'approprier et se construire poétiquement un destin en interaction étroite avec celui imaginé par son ou ses collègues. Les poètes sont donc amenés à négocier avec le hasard pour tenter de le déjouer. Il n’y a qu’à scruter leurs visages hagards et concentrés au cours des cinq minutes silencieuses qui suivent le tirage au sort pour deviner comment ils « envisagent » l'improvisation des thèmes. La gara oppose donc au jeu de hasard ou «alea $\gg$ du maju un jeu de compétition ou «agôn », selon la terminologie de Roger Caillois ${ }^{11}$.

Par rapport au jeu de Mai qui assigne des sorts en proférant une parole fixe et indiscutable avant de tirer les objets du panier, la joute procède de manière inverse : la parole improvisée des poètes arrive après le tirage des thèmes et opère sur eux un travail de questionnement, de déconstructionreconstruction, et finalement d'appropriation. Soit une manipulation poétique du thème et du sort.

La poésie dans le maju énonce le « tirage des sorts », alors que dans la gara poetica, elle négocie le «tirage au sort ». Cette différence se vérifie à travers la forme des réponses poétiques qui sont apportées au hasard dans les deux jeux.

\section{Poésie incantatoire/poésie oratoire}

Dans ces jeux, la confrontation avec le hasard se fait à travers un échange en poésie $^{12}$, une poésie chantée, et qui plus est avec un chœur (ou à plusieurs). Quel genre de poésie est à l'œuvre dans ces jeux avec le hasard ? Selon que le chant a lieu avant ou après l'action de tirer au sort, la poésie et le chant sont bien différents.

\section{L'incantation du maju}

S'agissant du maju, seule nous est parvenue la transcription des paroles poétiques chantées à des moments précis de la cérémonie. D’abord, pendant que les objets sont déposés dans le panier, une des jeunes filles présentes chante les vers suivants qui forment des quatrains (battorinas) prolongés dans le chant par un vers de variation ou de reprise : 
Un pigeon vrai/Descend du ciel/Avec une monnaie dans la bouche/Ouvrez la bourse/Afin de la jeter dedans.

Puis, une fois les objets déposés, d'autres vers sont chantés :

[Que] Christ bénisse/Sous terre la vigne/Et le blé en herbe/La vigne sous terre/ Et l'orge en épi/[Que] Christ bénisse.

Enfin, avant l'extraction du premier objet est entonnée par une jeune femme, par plusieurs personnes ensemble ou un chœur (selon les sources) une invocation au mois de mai (appelée Maia) ${ }^{13}$, et suivie d'un distique (duina) de bon augure ou de bénédiction (torrada bona) comme celui-ci :

Comme court l'eau de la mer/Ainsi puissent courir à vous les biens.

Avant le deuxième tirage au sort, la même invocation à Mai est suivie d'un distique de mauvais augure ou de malédiction (torrada mala) :

Comme court l'eau dans la plaine/Ainsi puissent courir vos entrailles derrière vous.

Cirese précise que le chant continue jusqu'à ce qu' il n'y ait plus d'objet dans le panier. Le caractère cérémoniel des paroles se perçoit à travers les expressions symboliques : « Pigeon vrai descend du ciel » renvoie au jet des objets dans le panier et «Christ bénisse » est un souhait de prospérité agricole au moment du printemps. Il en va de même dans l'invocation au mois de mai où le « coup de fouet » représente le coup du sort, et la contradiction « par amour » indique que ce mal est infligé pour un bien. Quant aux distiques qui énoncent les sorts, ils sont fixes mais peuvent être variés de façon canonique. Les formules métaphoriques (《l'eau de la mer $\gg, ~ «$ les entrailles $\gg$ ), les structures parallèles et répétées (« comme..., ainsi... »), le balancement du rythme et l'emploi du subjonctif injonctif (« puissent ») relèvent d'un langage magique pour « jeter » un sort. L'énonciation poétique précédant le tirage des objets, prédit ou plutôt fait advenir le sort qui est effectif dès lors qu'il est énoncé. Ici, la poésie est performative puisque « dire c'est faire $\gg^{14}$, et elle a une fonction incantatoire pour cette cérémonie de divination.

Même si aujourd'hui il ne reste pas de trace du chant, celui-ci est attesté par des témoignages et aussi par le nom même du jeu : Cantare su maju («chanter Mai »). Dans cette expression le verbe « chanter » prend le sens de $\ll$ célébrer $»$. 


\section{L'improvisation dans la gara}

Il en va autrement dans la gara poetica, puisque le hasard n'est pas entériné par une parole fixe de type incantatoire, mais combattu à travers la parole oratoire d'une joute improvisée. Pour relever le défi du tirage au sort, les poètes doivent défendre chacun leur thème contre celui du collègue en chantant une strophe à tour de rôle. Mais cette fois, l'alternance ne régule pas la distribution équitable des sorts bons et mauvais du maju ; elle est le mouvement dialectique d'une discussion sur des thèmes opposés. La poésie improvisée de la gara est discursive et sert un débat argumenté.

Ce débat est régi par des règles poétiques qui valent comme règles du jeu. Une contrainte métrique impose le huitain d'endécasyllabes rimés $(A B / A B$ ou BA / AB ou BA / CC). L'improvisation doit suivre des parcours obligés : celui du vers dont les schémas d'accentuation délimitent des patterns (patrons rythmiques) où vont se loger des formules prémémorisées ; celui de la strophe dont la construction logique comporte huit vers à fonctions différentes (deux vers de réponse au défi du collègue, deux vers pour détruire son argument, deux autres pour lui opposer un contre-argument et deux derniers vers de défi) ; enfin celui du thème qui doit être développé selon trois ordres combinés : chronologique, logique, et analogique ${ }^{15}$. Voici deux strophes d'une joute sur les thèmes : «la Langue » et «l'Épée », chantées dans les années cinquante par les poètes Raimondo Piras et Salvatore Tucconi.

\section{Raimondo Piras : « la Langue $》$}

Moïse a toutes les qualités

D'un homme politique, d'un homme d'état.

Avec sa langue il vante les vertus

Et fait de tout un examen critique.

Moïse a donné les commandements

Et ce n'était ni fantaisie ni mythe.

Il est un chef, il soigne et convertit,

Il sait prêcher, c'est pour ça qu'on le suit.
Mosè at totugantos sos acentos

De istatica 'e omine politicu Preigat cun sa limba sos talentos

E tenet in su totu esame criticu Mosè at dadu sos cumandamentos E non fit fabulosu ne fit miticu. Iss'est padronu, cunvertit e meigat, Li ponent fatu ca proite preigat.

Però Davide est su distintu rè Ch'at balanzadu totu sas bataglias. Deus agiuat puru a Giosuè A nd'imbolare sas setes muraglias. In tota Palestina ponet pè,

Durat su sole e bolant sas iscaglias. 
Dieu lui-même autorise l'épée

Et permet que de sang elle soit souillée. ${ }^{16}$
E finas Deus s'ispada l'amitit

E de l'insambenare già permitit.

Ces contraintes constituent des normes exigeantes mais sont aussi des aides à l'improvisation qui a recours en outre à toutes sortes de procédés et de stratégies : travail d'anticipation en constituant des stocks d'arguments, d'exemples, de rimes et de formules, qui seront mobilisés sur le moment et variés afin de s'adapter aux thèmes débattus ; stratégies logiques et paralogiques pour déstabiliser le collègue, comme les syllogismes dignes des sophistes, et le recours à l'art de la ruse (mètis) et de la réponse au moment opportun (kairos), qui signent le talent.

Enfin, dans la gara, la poésie est chantée sur une mélodie (tragiu) propre à chaque poète avec l'accompagnement d'un petit chœur polyphonique à trois voix (tenore) qui intervient entre les vers et les strophes en émettant, sur un accord parfait, des syllables sans signification (par exemple : «bim bam bom »). Le chœur donne ainsi du temps au poète pour improviser, et la mélodie lui permet d'inscrire ses vers en ajustant leur métrique grâce à la musique. En ce sens le chant aide à l'improvisation. Et cette fois le verbe cantare, employé pour la gara, signifie : « improviser ».

\section{La poésie mise en scène}

Ces jeux de type game en lien avec le hasard sont aussi des jeux de type play, au sens où ils se déroulent suivant une théâtralité particulière. Dans les deux cas, le play vient théâtraliser le game de manière indissociable, pour le bon fonctionnement du jeu et son efficacité. Ainsi, la consultation du hasard est donnée à voir et à entendre en public, et la poésie qui l'exprime se trouve placée sur une scène et comme «mise en scène » pour être partagée. Cela se fait suivant des codes qui opèrent une rupture avec le quotidien, installent un espace spécifique et une autre temporalité, mettent en place une action et des rôles, animent une corporalité.

\section{Espace et temps transformés}

Du point de vue du temps, ces jeux se déroulent tous deux au cours d'une fête qui a lieu à date fixe et revient de façon cyclique chaque année. À cette occasion, la convocation du hasard constitue un événement : le maju célèbre le renouveau de la nature au début du mois de mai et la gara poetica honore un saint patron lors d'une fête calendaire afin d'obtenir ses grâces pour le village. Leur déroulement aussi est balisé et rythmé. Dans le maju comme dans la gara, l'ordre et la durée des prises de parole s'établit selon une loi d'al- 
ternance : bon augure/mauvais augure et thème/thème opposé (identifiés d'ailleurs comme thème « fort » ou « faible $»)$. Ce balancement est rendu sensible par le rythme mesuré des strophes chantées (quatrains, distiques ou huitains). Dans le maju chaque strophe d'augure, chantée par un participant, est suivie par l'extraction d'un objet, et ainsi de suite. Dans la gara, chaque improvisateur écoute la strophe du collègue en même temps qu'il prépare la sienne avant de la chanter (en une minute environ). La dispute sur les thèmes compte une soixantaine de strophes et dure à peu près une heure, sur un rythme très régulier marqué par la diction syllabique des poètes, les interventions du chœur à chaque strophe et les coups sonnés tous les quarts d'heure au clocher de l'église qui se trouve à proximité.

Ces jeux se déroulent dans un espace et un décor particuliers, distincts du lieu de vie habituel. Ils délimitent une « scène » circulaire où les participants peuvent voir et être vus. Les joueurs du maju sont assis en cercle à même le sol, par exemple devant la porte d'une maison. Au-dessus de leurs têtes un drap blanc est dressé en baldaquin, comme pour isoler du ciel une telle distribution des sorts. Les poètes se tiennent, eux, sur une estrade surélevée par rapport à la place du village, et improvisent «à la volée » $(a$ bolu $)$ à ciel ouvert, par opposition au travail d'écriture qui a lieu entre quatre murs et un toit. Placés côte à côte, ils sont entourés à l'avant par le public et à l'arrière par le chœur et le comité. Au milieu de ces structures circulaires, le panier et la casquette d'où sont extraits les sorts et les thèmes constituent une sorte d'épicentre du hasard. Tout autour : le village et sa communauté.

\section{Le suspense comme action dramatique}

Ces consultations du hasard ne manquent pas de créer une attente et une certaine tension dramatique. Après chaque strophe de bon ou de mauvais augure, les participants au maju espèrent ou appréhendent en secret l'extraction de l'objet qui leur appartient. De même, au cours de la gara, la tension des poètes est palpable au moment du tirage au sort des thèmes puis à chaque strophe du collègue. Le public, quant à lui, ne perd pas une syllable de la joute, il est dans une continuelle attente et manifeste une grande attention. Dans les deux cas, la dramatisation produite par ces jeux avec le hasard constitue une intrigue à laquelle sont associés des jeux de rôles. En effet, dans le maju comme dans la gara, les sorts et les thèmes donnent lieu à une identification : celle des participants du maju à leurs sorts par le truchement magique des objets personnels; celle des poètes à leurs thèmes qu'ils s'approprient et appellent aussi « parte $\gg$ (rôle). Tout au long de la joute, ils les font d'ailleurs parler à la première personne dans une grande prosopopée : «Je suis l’Amour, 
tu es la Haine, et voici ce que je te dis... ». Cela est particulièrement sensible au cours des thèmes légers et drôles, qui donnent lieu à la construction d'un petit scénario dialogué, comme : Le «Marocchino » (Travailleur émigré moins souvent marocain que sénégalais) épousera-t-il la « Jeune fille » contre le consentement de son « Père » ?). D’autant que le dénouement de cette commedia dell'arte sarde, et la leçon qu'on saura en tirer, ne sont pas fixés à l'avance et dépendent du talent des improvisateurs.

\section{Le geste et la parole}

La gestuelle de ces jeux, étroitement associée aux paroles chantées, varie avec la nature du «spectacle » présenté. Dans le maju, les attitudes et gestes qui nous sont rapportés accompagnent ou suivent les paroles poétiques de façon décisive. Les participants s'assoient à même le sol en posture de soumission et les objets sont jetés dans le panier en même temps qu'une strophe est chantée pour donner à ce geste une valeur symbolique et sacrée. Puis, les objets sont retirés du panier un à un à la suite d'une strophe d'augure. La parole poétique et le chant chargent ces gestes d'efficacité et leur donnent du pouvoir - bénéfique ou maléfique - en scellant à travers eux le lien entre leur propriétaire et son sort.

Dans la gara, les poètes tirent au sort les papiers de leur thème. Au moment où ils « touchent » les papiers, où les thèmes leur « échoient» et sont «acceptés comme un devoir » (triple sens de «toccare $»$ ), ils sont assis. Mais ensuite ils se lèvent tour à tour pour improviser, dans une posture bien droite d'affrontement avec le hasard. Le bas du corps reste fixe et bien campé sur les jambes, alors que le haut du corps développe une gestuelle discrète d'orateur, en lien direct avec un discours qu'ils produisent dans le même temps qu'ils le profèrent. Les gestes des bras, des mains et des doigts accompagnent la parole et la font vivre : ils la pointent, la soulignent, la distribuent mais sans la figurer. Leur physionomie participe aussi du message, en particulier le sourire ironique qui vient souvent mettre en doute les propos. Toutefois, les poètes ne tombent jamais dans un jeu d'acteur : aucun contact physique entre eux, aucun regard échangé. Ils restent tournés vers le public et lui adressent le discours destiné à leur collègue, suivant un schéma triangulaire de la communication (poète-public-poète) qui diffère de celui du dialogue théâtral.

\section{Un « théâtre de soi »}

On le voit, ces jeux de la poésie et du hasard présentent certaines caractéristiques spectaculaires et polysensorielles. Mais le profond ancrage social, l'interrogation du hasard et les représentations symboliques proposées, en 
plus de l'importance qu'y joue la poésie, font de ces pratiques traditionnelles des objets anthropologiques complexes. Elles ne sont pas du théâtre et relèvent plutôt du rituel, un rituel de la parole, du geste et de la voix, strictement codifié, esthétique, mais également social, surnaturel et sacré.

\section{Un spectacle total}

Du point de vue social, ces jeux ont une fonction de cohésion : ils impliquent la communauté villageoise et sont moins des sujets de division que d'agrégation. Malgré la rivalité qu'ils comportent, ce sont des occasions de construire ensemble la culture et ses valeurs. Les sorts - bons et mauvais - sont équitablement répartis et acceptés par tous dans le maju; et les poètes qui s'affrontent à travers une dialectique dans la gara finissent par se réconcilier et proposer une synthèse de leurs thèmes opposés. Il n'est d'ailleurs pas indifférent que les deux pratiques comportent un chœur (ou se chantent à plusieurs), ce qui est une émanation à la fois musicale et sociale du groupe ou du village concernés. L'ensemble des jeunes gens du maju chantent collectivement l'invocation à Mai et attestent le consensus dans la distribution des sorts, au nom d'une croyance partagée. Le chœur du village mis à disposition pour la gara fait entendre un accord sonore, une forme de contrepoint acoustique du désaccord des poètes en joute.

Au-delà du social, ces jeux avec le hasard révèlent la conception qu'une société se fait du destin, et touchent par là-même au surnaturel et au sacré. Le maju est une cérémonie divinatoire collective et magique, qui a valeur de prédiction, et fonctionne tel un horoscope oral permettant a posteriori de lire les événements qui se produisant le long d'une année ; alors que la gara invite les poètes et le public à envisager a priori des destinées possibles avant d'agir. L'improvisation y est un don offert au saint patron de la fête en échange de ses grâces, et de ce fait la gara participe d'un échange à dimension sacrée.

\section{Une société mise en jeu}

Ces jeux auxquels prend part toute une communauté ne séparent pas radicalement acteurs et spectateurs. Au contraire, le public du maju et celui de la gara y sont pleinement actifs. Chacun des joueurs du maju est un protagoniste indispensable pour équilibrer la distribution des sorts. Il s'investit dans l'action, accepte l'aléa et consent au résultat. Dans la gara poetica, le public réagit au discours des poètes à travers des interactions discrètes mais constitutives de la joute, que les poètes peuvent percevoir. Il critique, approuve ou désapprouve, sourit ou fait la moue. Mais par son attention à chaque syllable prononcée, par sa connaissance des codes, son savoir partagé, son expertise 
et parfois son talent à faire des vers, il contribue d'une certaine manière à la réalisation de la joute. À la fin de la gara, c'est lui qui l'évalue sur la place du village, à travers une autre petite joute ouverte où chacun défend son champion à coups de citations mémorisées comme autant d'éléments d'une mémoire collective. Le public est donc partie prenante, acteur et co-énonciateur de la gara comme du maju, qui sont réalisés avec lui et pour lui à travers la production d'une poésie partagée.

Le plus intéressant est sans doute qu'à l'occasion de ces jeux poétiques avec le hasard, la société se mobilise pour s'observer elle-même et se mettre en question. Cette prise de risque est sensible à travers la notion d'aléa : la plongée des mains dans le panier ou dans la casquette pour interroger le hasard, ou encore les images poétiques du « pigeon » dans le maju et de la «Déesse aux yeux bandés $\gg$ dans la gara. Elle met à l'épreuve sa connaissance, son talent, ses valeurs, son fonctionnement et sa solidarité afin de mieux préserver son unité. Elle se met en scène et en jeu dans un «théâtre de soi » solidaire et salutaire.

Cette situation n'est pas sans rappeler le rêve de Rousseau dans La Lettre à D'Alembert sur les spectacles ${ }^{17}$ où il critique « ces spectacles exclusifs qui renferment tristement un petit nombre de gens dans un antre obscur ; qui les tiennent craintifs et immobiles dans le silence et l'inaction $\gg$ et propose à la place un spectacle populaire, collectif et festif, quelque chose comme un théâtre de soi : « Plantez au milieu d'une place un piquet couronné de fleurs, rassemblez-y le peuple, et vous aurez une fête. Faites mieux encore : donnez les spectateurs en spectacle, rendez-les acteurs eux-mêmes ; faites que chacun se voie et s'aime dans les autres, afin que tous en soient mieux unis. »

\section{Le « spectacle » revisité}

Ces jeux sont donc des pratiques moins spectaculaires que spéculaires. Ils ne sont pas des représentations théâtrales mais les productions collectives d'une action, en l'occurrence la confrontation du hasard et de la poésie, une poésie performée et en acte ${ }^{18}$.

En fin de compte l'appellation « spectacle » utilisée pour ces jeux au début de cette étude se trouve remise en question. S'il y a « spectacle » cela ne peut pas être compris comme la représentation d'une action fictive comprenant une intrigue prédéfinie, mise en scène et menée par des acteurs devant des spectateurs qui y assistent passivement. Il faut plutôt comprendre cette notion à partir de l'étymologie latine de «spectare » qui signifie « assister à $», \ll$ être en présence $»^{19}$. Cela souligne l'importance du fait de prendre 
part, d'être ensemble pour construire et partager un même événement qui est en train de se faire.

C'est donc autre chose qui se joue là. Et s'il y a « représentation », il ne s'agit pas d'une figuration mimétique fixée au préalable et répétée à l'identique, mais bien d'un «re-enacting » au sens que lui donne Jack Goody ${ }^{20}$, soit de la ré-activation ou re-mise en action d'une forme-structure vive, celle du maju ou de la gara, qui grâce à un aléa générateur fait advenir à chaque fois une autre production, un événement nouveau.

On voit bien qu'en Sardaigne, comme dans d'autres sociétés de tradition orale, jeu et théâtre entrent en rapport. Ces jeux poétiques avec le hasard que sont le maju et la gara présentent à l'intérieur du game un certain play, mais qui est mis à distance et en abyme : le jeu se donne en spectacle, ce qui peut être condensé dans la formule : to play the game.

Plus exactement, c'est la société qui se représente elle-même. En jouant avec le hasard au moyen de la poésie, elle s'implique collectivement et engage sa conception de la vie et du destin, qui est sans cesse remise en cause. Ainsi la société sarde se pose la question d'accepter le hasard ou d'agir sur lui. Et plus largement elle se demande quelle attitude avoir devant l'aléa : soumission ou action ? Elle y répond de deux manières distinctes : la joute poétique improvisée qui affonte le hasard et a encore cours aujourd'hui, ou la cérémonie divinatoire de soumission au hasard, qui n'est plus pratiquée.

Finalement, ce qui se passe à ces occasions n'est pas exactement du théâtre, mais une action collective produite conjointement par le hasard et la poésie, créant à chaque fois un nouvel événement. On pourrait parler de jeux à «fonction créatrice de culture », selon l'expression de Johan Huizinga ${ }^{21}$, qui sont tout à fait indispensables à la vie (ou la survie) de ces petites sociétés de tradition orale que l'on peut encore observer aujourd'hui.

\section{Notes}

1. Théâtre : «Art dont le but est de produire des représentations (régies par certaines conventions) devant un public, de donner à voir, à entendre une suite d'événements, d'actions, par le biais d'acteurs qui se déplacent sur la scène et qui utilisent ou peuvent utiliser le discours, l'expression corporelle, la musique. » Dictionnaire Trésor de la langue française électronique (TLFI), 1971-1994.

2. Matteo Madao, Armonie dei Sardi, Cagliari, Reale Stamperia, 1787.

3. Alberto de La Marmora, Voyage en Sardaigne, vol. I, 2e édition, Paris, 1839, p. 265-267. 
4. Filippo Valla, "Il primo maggio in Ozieri ", Archivio per lo studio delle Tradizioni popolari, XIV, 1895, p. 525-527.

5. Rosa Losengo a recueilli des témoignages de terrain en 1960 pour les besoins d'une recherche d'Alberto Mario Cirese, qui a donné lieu à un article paru en 1964.

6. Alberto Mario Cirese, «Essai d'analyse d'un jeu cérémoniel du $1^{\text {er }}$ mai en Sardaigne : cantare su maju ", Actes du VTe Congrès international des sciences anthropologique et ethnologiques, Paris, 30 juillet-6 août 1960, tome II, Ethnologie, Musée de l'Homme, 1964.

7. Ibidem.

8. Alberto Mario Cirese, «Du jeu d'Ozieri au numerus clausus des Bienheureux de Dante. Essai d'une typologie idéologique », L’Homme, vol. 35, n 136, oct.-déc. 1995, p. 95-112.

9. Maria,Manca, La Poésie pour répondre au hasard. Une approche anthropologique des joutes poétiques de Sardaigne, Paris, Éd. MSH/CNRS, 2009.

10. Il existe sous différentes formes dans de nombreuses cultures du monde: en Espagne (trovo), aux Baléares (glossa), en Corse (chjami e rispondi), mais aussi au Brésil (desafio), etc.

11. Roger Caillois, Les jeux et les hommes. Le masque et le vertige, Paris, Gallimard, 1967 [1958].

12. Le terme de «poésie » est à prendre ici dans un sens large qui inclut les dimensions musicale et pragmatique de cette pratique. Sa signification rejoint celle du mot grec mousikê ou " domaine des Muses ». Voir Claude Calame, Florence Dupont, Bernard Lortat-Jacob et Maria Manca, La Voix actée. Pour une nouvelle ethnopoétique, Paris, Kimé, 2010, p. 7.

13. Il est mai, il est mai / Et [que] vienne le bien / Avec tout le soleil d'amour / Plus belle est la fleur / Que la petite marguerite / Un coup de fouet [ou une botte d'épines]: Quion te le donne par amour.

14. John L. Austin, Quand dire, c'est faire, Paris, Seuil, 1970.

15. Lordre chronologique impose de citer les exemples anciens avant les modernes, l'ordre logique appelle une discussion des arguments et lordre analogique demande que chaque poète cite dans ses strophes le nom de son thème et reprenne dans la strophe précédente du collègue un mot sur lequel il rebondit. Voir Manca, 2009.

16. Voici deux autres strophes plus amusantes d'une joute sur les thèmes : «la Loi » et le "Hors-la-loi », chantées à Pozzomaggiore en juillet 1975 par les poètes Raimondo Piras et Peppe Sotgiu :

\section{Raimondo Piras : «le Hors-la-loi ”}

Il est vrai que tu n'as pas de chance

Et tu acceptes tout ce quion te fait

Malheureux qui ne jouit pas de faveurs

Car on le traite comme une bête de somme.

Moi je m'avance avec un pistolet

Et je dis : "Ouvrez ce coffre-fort! "

Et eux, en tremblant de concert

Ils l'ouvrent et me donnent l'argent.

Peppe Sotgiu : « la Loi »

Moi je ne profère pas mille menaces
Zertu sa tua est una malasorte $E$ de su chi ti faghent ti consola. Iscuru a chie no at favore in corte Lu tratana che bestia 'e mola. Deo bi ando cun-d-una pistola E naro: "Aberi cussa cascia-forte!". $E$ issos tremulende totu umpari L'aberint e m'intregant su dinari.

Deo non b'ando cun milli minetas, 
Toi tu préfères être un hors-la-loi.

Et tu leur dis : "Ouvrez-moi le coffre", Autrement tu les jettes par le balcon.

Mais un jour les carabiniers

Te mettront les menottes aux poignets,

Et ce que tu auras sorti du coffre

Tu devras le payer en prison.
Tue sa prepotenzia preferis.

E li naras : "O sa cascia mi aberis». Si no da su balcone che lu 'etas. Ma chalchi die sos carabineris T'ant a poner in brussos sas manetas, E su chi chogas dae su cascione Iscontare lu deves in presone.

17. Rousseau, Lettre à d'Alembert sur les spectacles, Paris, Gallimard, 1992 [1758].

18. Voir Claude Calame, Florence Dupont, Bernard Lortat-Jacob et Maria Manca, La Voix actée. Pour une nouvelle ethnopoétique, Paris, Kimé, 2010.

19. Une journée d'étude intitulée : "Spectateurs grecs et romains. Corps, modalités de présence, régimes d'attention " a été organisée par Emmanuelle Valette et Stéphanie Wyler, de Paris Diderot, les 4 et 5 novembre 2016 à l'Université Paris Diderot et à I'INHA.

20. Jack Goody, Representations and Contradictions, Oxford, Blackwell Publishers, 1997.

21. Johan Huizinga, Homo ludens. Essai sur la fonction sociale du jeu, Paris, Gallimard, 1995. 